ich für eine gesetzliche Regelung zur Steigerung des Anteils der Frauen in Führungspositionen, plädiere ich für eine gesetzlich geregelte Frauenquote - zumindest vorübergehend.

Ich bin fest davon überzeugt, dass wir spätestens in der nächsten Legislaturperiode und mit Unterstützung aus der EU den Weg der Freiwilligkeit und damit auch des Schneckentempos bei der Realisierung der Frauenquote verlassen werden. Und ich bin davon überzeugt, dass wir dann in
Deutschland endlich realisieren werden, dass eine gesetzliche Frauenquote nicht nur verfassungsrechtlich möglich, sondern auch wirtschaftlich sinnvoll ist. Vielleicht ergeht es der Frauenquote in Zukunft dann auch einmal so wie der Gleichberechtigung von Mann und Frau, nämlich, dass wir sie als Selbstverständlichkeit empfinden. Ich jedenfalls, würde es ihr, unserer Gesellschaft und auch unseren Wirtschaftunternehmen wünschen.

\title{
Zähne für den Tiger!
}

\section{Sigrun Mast, Maître en Droit}

Rechtsanwältin, Hamburg

Auch ich begrüße Sie herzlich zu „Wie soll der Wandel gelingen “. Ich heiße Sigrun Mast, bin Mitglied im Deutschen Juristinnenbund und als Rechtsanwältin in der Wirtschaftsprüfungsgesellschaft RöverBrönnerSusat tätig.

Birgit Kersten, die maßgeblich beim Deutschen Juristinnenbund das Projekt „Aktionärinnen fordern ein “ begleitet, hat mich im Anschluss an einen internen Workshop gefragt, ob ich im Rahmen einer Veranstaltung ein kurzes Statement abgeben könne. Anlass war insbesondere mein Besuch des Euroforum-Seminars „Der zertifizierte Aufsichtsrat“ in 2011.

Das Seminar „Der zertifizierte Aufsichtsrat“ ist ein sechstägiges Seminar. Es gibt einige Übereinstimmungen mit dem Projekt des Verbands deutscher Unternehmerinnen „Wahrnehmung von Mandaten in Aufsichtsgremien“. Beim Euroforum gibt es aufgrund der anderen Zielgruppe kein Kommunikations- und Medientraining. Vielmehr ist der rechtliche Schwerpunkt verstärkt. Zusätzlich existieren ein ausführliches bilanzielles Modul und ein Abschlusstest. Nun, in allen wesentlichen Punkten stimmen beide Seminare überein. Allerdings hat sich Birgit Kersten in einer weiteren Mail gewünscht, dass ich die Frage „Wie soll der Wandel gelingen?“ aus meinem Arbeitsbereich und meiner persönlichen Sicht beantworte. Ok. Zunächst: Wo stehen wir?

Norwegen hat bereits eine Quote von 40 Prozent für Frauen in Aufsichtsräten eingeführt - norwegischen Aufsichtsräten. Allerdings kontrollieren norwegische Aufsichtsräte die Geschäftsführung nicht nur, sondern nehmen Geschäftsführungsaufgaben wahr. Deutsche Aufsichtsräte sind Überwachungsorgane. Wenn wir über einen Wandel reden, müssen wir uns fragen, ob wir einen norwegischen Wandel wollen; dann heißt es: Frauen in Führungspositionen. In Norwegen ist dieser rechtlichen Umsetzung eine hitzige gesellschaftliche Diskussion vorangegangen. Wir stecken mitten in dieser gesellschaftlichen Diskussion. Die gesellschaftliche Forderung ist die nach einer gesetzlichen Quote für Frauen in Aufsichtsräten.

Doch wie hängen Gesellschaft und Recht zusammen? Teils prägt die Gesellschaft das Recht, teils prägt das Recht die Gesellschaft. Für eine umfassende gesetzliche Änderung bedarf es einer weitgehenden gesellschaftlichen Akzeptanz. Diese fußt auf einer Notwendigkeit des Wandels - dem Sollen. Eine Mehrheit muss die Konsequenzen, die hieraus erwachsen, auch wollen. Die demografische Entwicklung und der daraus resultierende Fachkräftemangel führen zu einer solchen Notwendigkeit des Wandels. Glauben wir aktuellen Umfragen bejahen immer mehr Deutsche eine Frauenquote für Führungspositionen. Diese stellt einen Teil des Wandels dar. Meine weiteren Ausführungen beziehen daher sowohl das aktuelle Recht als auch das gesellschaftliche Sein, Wollen und Sollen mit ein.

Nehmen wir zunächst das praktische Beispiel des Seminars „Der zertifizierte Aufsichtsrat“. Wer besucht das „neue“ Seminar „Der zertifizierte Aufsichtsrat“ und warum? Das Seminar wird von Aufsichtsräten und Beiräten, von Geschäftsführern, Vorständen und internen und externen Beratern besucht. Es war ein kleiner Kreis von knapp über zehn Teilnehmern, davon drei Frauen. Alle interessierte die aktuelle Rechtslage. Einige hatten praktische Fragen aus dem Alltag. Aufsichtsräte und deren Berater sind seit der Wirtschaftskrise an diesen Seminaren interessiert. Die Frage der Aufsichtsratshaftung spielt eine immer stärkere Rolle. Spätestens seit dem Urteil im Fall ARAG-Garmenbeck. Danach muss der Aufsichtsrat Schadensersatzansprüche gegen den Vorstand verfolgen, wenn er nach eigener Prüfung von einer Schadenersatzpflicht des Vorstands ausgeht. Verstärkt fragten die Teilnehmer auch nach den wichtigsten Konditionen für eine DirectorsAndOfficers-Versicherung. Ein Vorstandsmitglied gab zu, dass seine Aufsichtsräte verstärkt Fragen stellen, bei denen er sich fragt, ob sie diese Fragen stellen müssen und dürfen.

Inwieweit ist der "Wandel“ bei diesem Seminar Thema? Rechtlich wird der Wandel durch den Deutschen Corporate Governance Kodex auf Ebene der Besetzung von Führungspositionen und des Aufsichtsrates thematisiert. Bei börsennotierten Gesellschaften erklären Vorstand und Aufsichtsrat jährlich, ob den Empfehlungen des Deutschen Corporate Governance Kodexes entsprochen wurde oder welche Empfehlungen nicht angewendet wurden oder werden und warum nicht. Der Deutsche Corporate Governance Kodex 
verpflichtet zum einen den Vorstand bei der Besetzung von Führungsfunktionen im Unternehmen auf Vielfalt, „Diversity “, zu achten und dabei insbesondere eine angemessene Berücksichtigung von Frauen anzustreben. Der Aufsichtsrat soll für seine Zusammensetzung konkrete Ziele benennen, (...) die insbesondere eine angemessene Beteiligung von Frauen vorsehen sollen. Trotz der eher weichen Formulierung müssen nach der Rechtsprechung zur Umsetzung der Empfehlungen des Kodex konkrete Ziele umschrieben werden. Eine allgemeine Formulierung wie „Beteiligung von Frauen und Ausländern “ wäre dann keine konkrete Zielbenennung. Und wenn die Empfehlungen nicht beachtet werden? Eine fehlerhafte Entsprechenserklärung kann zur Anfechtbarkeit der Entlastungsentscheidung des Vorstands führen.

Kommen wir zur gesellschaftspolitischen Ebene: Auch die Frauenquote in Aufsichtsräten und die Empfehlungen des Kodexes haben zwei Dozenten kritisch angesprochen. Der eine Dozent hat nach einem Blick in die gemischte Runde lediglich den Diskussionsstand kurz skizziert. Der zweite Dozent hat mit Blick auf den Boden ein wenig über den geplanten hinderlichen Eingriff in unternehmerische Freiheiten geschimpft. Dies hat mich nicht ansatzweise geärgert. Warum? Die Reaktion der anwesenden weiblichen und männlichen Seminarteilnehmer war ein amüsiertes Lächeln.

Inwieweit ist der Wandel in der Praxis vorangeschritten? Dazu möchte ich noch einmal auf die Frage zurückkommen: Wo stehen wir? Nun, ich war 2010 im Rahmen des Projektes „Aktionärinnen fordern ein“ bei der Hauptversammlung der Volkswagen Aktiengesellschaft. Unsere Aktion zeigt Wirkung. Ferdinand Piëch möchte demnächst auf Anteilseignerseite eine Frau im Aufsichtsrat der Volkswagen Aktiengesellschaft sehen - seine Frau. Sie hat eine Ausbildung zur Kindergärtnerin. Durch jahrelanges „Training on the job“ hält sie sich der Aufsichtsratsposition für gewachsen. Der Deutsche Corporate Governance Kodex spricht von erforderlichen Kenntnissen, Fähigkeiten und Erfahrungen. Warum nicht? Nach Friede Springer und Liz Mohn kommt nun Ursula Piëch. Hätte eine dieser drei Frauen nach den Kriterien, die Frauen und Frauenverbände aufstellen, eine Chance auf ihre jeweilige Position gehabt?

Viele Unternehmen gehen neue Wege im Personalbereich. Beispielsweise hatten 2010 mehr als 40 Prozent der DAXUnternehmen das Audit „Beruf und Familie“ erworben. Dieses Zertifikat und ähnliche Werkzeuge zur Familienfreundlichkeit fragen verstärkt auch mittelständische Unternehmen nach. Auch mein Unternehmen.

Wie kann der Wandel gelingen? Nach meiner Überzeugung ist nicht die Frage, ob der Wandel gelingt. Wir stecken mittendrin. Wichtiger ist es daher zu fragen, wie der Wandel gut gelingt. Gesellschaftlich wird die demografische Entwicklung unserer Gesellschaft dazu führen, dass ohne Frauen wirtschaftliche Entwicklung nicht denkbar ist. Nun könnten wir abwarten, bis auch die letzten Dinosaurier in der Wirtschaft und Politik Frauen wollen, weil sie Frauen brauchen. Einsichtige Menschen sind von sich aus bereit, verbesserte
Bedingungen zu schaffen. Mir persönlich reicht es, wenn ein Großteil einsichtig ist und einen Wandel unterstützt. In diesem Moment kann beispielsweise eine Quote katalysierend wirken.

Auf Ebene der Führungskräfte werden wir nach den Erfahrungen des vergangenen Jahrzehntes nur mit einer Quote einen echten Wandel gewährleisten können. Ich bedauere den Eingriff in unternehmerische Freiheiten. Die Einführung der Quote sollte meines Erachtens zurückhaltend erfolgen. Quote ja, aber zeitlich in Übereinstimmung mit den Wahlzyklen und Regelungen des Aktiengesetzes. Zurückhaltung aber nicht in Hinblick auf Konsequenzen bei Nichteinhaltung der Quote.

Unabhängig von der Quote müssen Frauen den Wandel wollen. Eine Frau will ihre persönliche berufliche Karriere, wenn der Job stimmt, das Gehalt und das Umfeld. Für die Entscheidung für den Job sind Frauen selbst verantwortlich. Zunächst zum Gehalt: Der Gender Pay Gap kann von staatlicher Seite durch die Einführung echter Lohntransparenz vermindert werden. Es gibt seit 2009 das Verfahren Logib-D zur Ermittlung der Lohngleichheit zwischen Männern und Frauen. Unabhängig von der Frage der inhaltlichen Überarbeitung von Logib-D kann das bislang freiwillige Verfahren mit Sanktionen versehen werden. In der Schweiz, die dieses Verfahren entwickelt hat, kann sich um öffentliche Aufträge nur bewerben, wer den Nachweis der Entgeltgleichheit erbringt. Aber können Frauen nicht auch nach AGG klagen? Können sie. Sie tragen dann die Prozesskosten, das Prozessrisiko und das Risiko der Stigmatisierung. Und sollte nicht auch der Staat dafür sorgen, dass Gleiches gleich behandelt, also hier bezahlt wird?

Zum Umfeld: Eine Quote wird schwierig umzusetzen sein, wenn nur Singlefrauen oder Frauen ohne Kinder in der Lage sind, die Anforderungen der Führungspositionen zu erfüllen. Seit Beginn des letzten Jahres gibt es eine „Charta für familienfreundlichere Arbeitszeiten“. Diese haben Bundeskanzlerin Angela Merkel, Familienministerin Kristina Schröder und Spitzenvertreter der Wirtschaft, Verbände und Gewerkschaften unterzeichnet. Oft genanntes Beispiel ist der Slogan „Kein Meeting nach 17 Uhr“. Kennen Sie diese Charta? Haben Sie deren Umsetzung seit einem Jahr beobachten können? Ich nicht. Wenn ich nicht gelesen hätte, dass es diese Charta gibt, hätte ich es nicht gemerkt.

In der Bevölkerung und der Wirtschaft gibt es eine wachsende Akzeptanz für den Wandel. Es gibt bereits viele gute Ansätze. Die Regelungen des Deutschen Corporate Governance Kodexes, Logib-D und die Selbstverpflichtung zur „Charta für familienfreundlichere Arbeitszeiten“ sind einige gute Initiativen. Derzeit sind sie nur in Form von Empfehlungen und Bekundungen existent. Bislang haben wir einen zahnlosen Tiger. Geben wir dem Tiger Zähne. Quote ja. Mir sind mindestens genauso wichtig die Änderungen im Arbeitsumfeld und in der Entlohnung.

Soweit meine persönliche Stellungnahme - und dies ganz ohne mein Lieblingsthema der flächendeckenden und qualitativ guten Kinderbetreuung zu erwähnen. Ich danke Ihnen. 